\title{
Uma Estratégia de Gerência de Configuração de Ativos de Processos de Software Apoiada por um Ambiente de Engenharia de Software Centrado em Processos
}

\author{
Gleison Santos, Mariano Montoni, Sávio Figueiredo, \\ Leonardo Orenstein, Ana Regina Rocha
}

COPPE/UFRJ - Universidade Federal do Rio de Janeiro

Programa de Engenharia de Sistemas e Computação

Av. Horácio Macedo, 2030, Prédio do Centro de Tecnologia, Bloco H, Sala 319

Caixa Postal 68511 - CEP 21941-914 - Rio de Janeiro, RJ

gleison,mmontoni, savio,darocha@cos.ufrj.br

\begin{abstract}
Products derived from software processes improvement projects must be managed, as well as in software development. Software processes are important organization's process assets and their evolution must be managed. This paper presents the process assets configuration management approach being used on Taba Workstation. Besides, the deployment of the approach in a software organization during the preparation to the MPS.BR appraisal is detailed.
\end{abstract}

Resumo. Assim como no desenvolvimento de software, os produtos derivados de projetos de melhoria de processos de software também precisam ser gerenciados. Os processos de software constituem importantes ativos de processo de uma organização e devem ter sua evolução controlada. Este artigo apresenta a abordagem de gerência de configuração de ativos de processo em uso na Estação Taba e descreve, também, o uso desta abordagem em uma empresa de software durante a preparação para uma avaliação seguindo o modelo MPS.BR.

\section{Introdução}

Iniciativas de definição, a execução e a melhoria de processos de software podem se beneficiar da existência e gerência adequada de ativos de processo, ou seja, artefatos que sejam considerados úteis para atender às necessidades de negócio da organização. Dessa forma, um ativo de processo pode ser definido como algo que a organização considere útil para atingir os objetivos de seus processos [Chrissis et al. 2006]. Processos de software são, obviamente, parte do conjunto de ativos de processos de uma organização. Outros exemplos incluem roteiros de documentação, itens de conhecimento (como diretrizes, lições aprendidas, melhores práticas etc.), ferramentas, materiais de treinamento etc. Uma base responsável pela gerência de tais ativos, geralmente denominada biblioteca de ativos de processos, deve ser capaz de armazenar os documentos considerados relevantes, garantir que eles sejam disponibilizados aos interessados e garantir que suas evoluções sejam controladas através de métodos apropriados de gerência de configuração e de garantia da qualidade. 
Modelos de maturidade como o CMMI [Chrissis et al. 2006] e o MPS.BR [SOFTEX 2007b] prevêem a necessidade de uma biblioteca de ativos de processo organizacional para permitir que os processos padrão da organização sejam consultados e recuperados, bem como os demais ativos de processo organizacional, necessários para adaptar os processos padrão e, assim, definir os processos para projetos específicos e para implementar o processo [SOFTEX 2007a].

Um dos fatores de sucesso relacionados à execução de iniciativas de melhoria de processos é a definição de uma infra-estrutura adequada [Baddoo e Hall 2003] [Niazi et al. 2005] [Santos et al. 2007]. A maior parte das organizações com baixos níveis de maturidade no desenvolvimento, no entanto, não possui uma infra-estrutura adequada para iniciar a iniciativa de melhoria de processos de software [Santos et al. 2007]. Podese dizer que há dois tipos de infra-estrutura de apoio à atividades relacionadas a processos e para sustentar ações de melhoria [Zaharan 1998]: (i) organizacional e gerencial, e (ii) técnica. Uma biblioteca de ativos de processos se encaixa nesta segunda categoria e deve conter mecanismos para apoiar os processos em uso e estar integrada às funcionalidades existentes para a definição, execução e melhoria de processos de software. Deve, também, possuir mecanismos que garantam a gerência de configuração dos ativos de processo, principalmente os relacionados à definição e melhoria dos processos de software para garantir um controle efetivo da evolução de tais ativos.

Dessa forma, o objetivo deste artigo é apresentar a estratégia para gerência de configuração de ativos de processos de software implementada na Estação Taba e adequada aos requisitos previstos no MPS.BR e CMMI. A próxima seção apresenta uma breve revisão da literatura sobre gerência de configuração de ativos de processos de software. A Seção 3 apresenta as principais características da abordagem proposta enquanto a Seção 4 apresenta algumas abordagens encontradas na literatura. A Seção 5 descreve um estudo de caso referente a utilização da abordagem proposta com o auxílio da Estação Taba. Por fim, a Seção 6 descreve perspectivas futuras de melhoria da abordagem e a Seção 7 apresenta as considerações finais.

\section{Gerência de Configuração de Ativos de Processos de Software}

Ao longo do ciclo de vida de um projeto de software uma grande quantidade de itens de informação é produzida e muitos desses itens provavelmente sofrerão alguma modificação ao longo do projeto devido a diversas causas (por exemplo, mudanças nos requisitos, correção de defeitos). Para que não haja inconsistência nos itens de informação importantes, a criação e as alterações desses itens devem ser acompanhadas e controladas [Rocha et al. 2001]. De uma forma geral, Gerência de Configuração pode ser definida como uma "disciplina que visa identificar e documentar as características de itens de configuração, controlar suas alterações, armazenar e relatar as modificações aos interessados e garantir que foi feito o que deveria ter sido feito" [IEEE 1990]. O foco está no controle rigoroso dos aspectos gerenciais e técnicos dos produtos de trabalho, incluindo o sistema entregue [Chrissis et al. 2006]. Restrições e guias relativas a Gerência de Configuração podem ser derivadas de várias fontes. Políticas e procedimentos definidos no nível corporativo ou organizacional podem influenciar ou definir as regras para projeto e implementação do processo de Gerência de Configuração para um determinado projeto. Da mesma forma, o contrato entre o adquirente e o fornecedor pode conter regras que afetem o processo de gerência de Configuração. Por exemplo, auditorias de gerência de configuração podem ser 
requeridas, ou pode ser necessário que alguns itens sejam colocados sob gerência de configuração [SWEBOK 2008].

Assim como no desenvolvimento de software, os produtos derivados de projetos de melhoria de processos de software também precisam ser gerenciados. Dessa forma, os itens sob gerência de configuração não devem se limitar aos itens criados e/ou utilizados em projetos, mas também a outros ativos de processo da organização, como padrões, procedimentos, descrições de processos e bibliotecas de reuso [Chrissis et al. 2006]. Dessa forma, no contexto deste artigo, o enfoque principal é na gerência de configuração dos ativos de processo. Os itens que devem compor a biblioteca de ativos de processo organizacional devem estar documentados, bem como os procedimentos de consulta e recuperação. É importante considerar que os ativos de processo organizacional são continuamente melhorados e, portanto, mecanismos de gerência de configuração dos itens que compõem a biblioteca devem estar definidos e implementados para permitir, por exemplo, que projetos sejam executados utilizando diferentes linhas base de ativos de processo organizacional [SOFTEX 2007a].

\section{Estratégia de Gerência de Configuração de Ativos de Processos de Software na Estação TABA}

\subsection{A Estação Taba e o Nível E do MPS.BR}

Desde 2003, a Estação TABA vem sendo utilizada pela indústria no apoio à implantação de processos com foco no MPS.BR e no CMMI. O principal objetivo da Estação TABA neste sentido é prover uma infra-estrutura consistente com os requisitos (ou parte dos requisitos) destes modelos para superar uma das dificuldades da implantação de iniciativas de melhoria de processos de software, que é a falta de recursos financeiros. A Estação TABA também provê a infra-estrutura para 0 desenvolvimento e integração de ferramentas de apoio à execução de processos de software. Os resultados de uso da Estação Taba foram considerados fatores determinantes no processo de obtenção de avaliações CMMI e MPS.BR em diversas empresas brasileiras. Como dados quantitativos, pode-se mencionar a redução do tempo gasto em retrabalho no projeto de $44 \%$ para $7 \%$ decorrente de iniciativa de melhoria de processo, realizada com o uso de ferramentas de apoio da Estação TABA, em uma destas empresas [Ferreira et al. 2006] [Ferreira et al. 2007].

A infra-estrutura atual da Estação Taba possui um repositório contendo informações do projeto de software coletadas ao longo do seu ciclo de vida e provê ferramentas de apoio aos níveis G, F, E, D e C do MR-MPS (e equivalentes do CMMI) [Montoni et al. 2007]. Atualmente, há teses de doutorado em andamento que visam a definição de ferramentas (e, possivelmente, alterações em ferramentas existentes) para a adequação aos níveis A e B do MR-MPS (e equivalentes do CMMI).

As tarefas relacionadas à definição de processos são implementadas através de uma ferramenta [Villela 2004] [VILLELA et al. 2004] que utiliza a norma ISO/IEC 12207 [ISO/IEC-12207 1998] [ISO/IEC-12207:Amd1 2002] [ISO/IEC-12207:Amd2 2004] como base para definição de processos e também modelos de maturidade como o MR-MPS e o CMMI. Os processos padrão e especializados para um determinado paradigma (por exemplo, estruturado ou orientado a objetos) são os processos padrão organizacionais. Para que o processo padrão seja utilizado num projeto específico, um 
processo especializado escolhido devido à adequabilidade ao projeto deve ser instanciado considerando as características do projeto (por exemplo, tamanho, complexidade do produto e características relevantes de garantia da qualidade e da equipe de desenvolvimento).

A instanciação do processo é apoiada por outra ferramenta [Berger 2003] [Villela et al. 2004] que permite a seleção do modelo de ciclo de vida e os métodos e ferramentas de desenvolvimento. A abordagem garante que os projetos sejam executados com base na definição de um processo adaptado a partir do conjunto de processos padrão da organização e facilitam a institucionalização de processos padrão. Outra ferramenta [Andrade 2005] [Campos et al. 2006] permite a avaliação e melhoria do processo de software organizacional e dos ativos de processo com base na avaliação de medidas de monitoração do processo, relatórios à gerência de alto nível gerados durante os projetos, checklists de aderência ao processo e análises post mortem e a revisão de lições aprendidas capturadas. Outra ferramenta [Silva Filho 2006] permite a identificação, planejamento, execução e avaliação das oportunidades de melhoria do processo através de projetos piloto.

O apoio à Gerência de Conhecimento é feito através da integração dos ambientes e todas as suas ferramentas a uma ferramenta de gerência de conhecimento [Montoni 2003] que apóia a disseminação das lições aprendidas e também a evolução do repositório de conhecimento com experiências adquiridas durante os projetos de software por todos os seus participantes. Esta abordagem permite a identificação de pontos fracos e fortes dos processos e também facilita a identificação de oportunidades de melhoria [Montoni et al. 2006]. Além disso, a maioria das ferramentas provê conhecimento específico e especializado sobre a subárea de Engenharia de Software relacionada, por exemplo, a ferramenta de adaptação de processo provê conhecimento sobre diferentes modelos de ciclo de vida. Outra ferramenta permite a descrição e visualização de estruturas organizacionais, englobando os profissionais alocados e as competências requeridas e possuídas ao longo dessas estruturas [Santos 2003] [Santos et al. 2005] construindo um mapa de conhecimento associado com uma "página amarela" de especialistas dentro da organização.

A existência de uma Biblioteca de Ativos de Processo para organização integrada à ferramenta de Gerência de Configuração possibilita o controle da evolução e divulgação destes ativos. As tarefas relacionadas ao processo de gerência de configuração são apoiadas pela ferramenta GConf [Figueiredo et al. 2004] que provê um mecanismo para monitorar as alterações nos requisitos ao longo do projeto, para controlar os pedidos de mudanças e para o versionamento de documentos e estabelecimento e liberação de baselines.

\subsection{Gerência de Ativos de Processo com Apoio de Gerência de Configuração na Estação Taba}

A estratégia utilizada na Estação Taba para a gerência de ativos de processo envolve a utilização de duas ferramentas integradas, uma para o cadastro e atualização dos documentos que compõem os ativos de processo organizacionais e outra a gerência de configuração destes ativos. Estas ferramentas, em conjunto, utilizam um processo cujo esquema pode ser visto na Figura 1. 


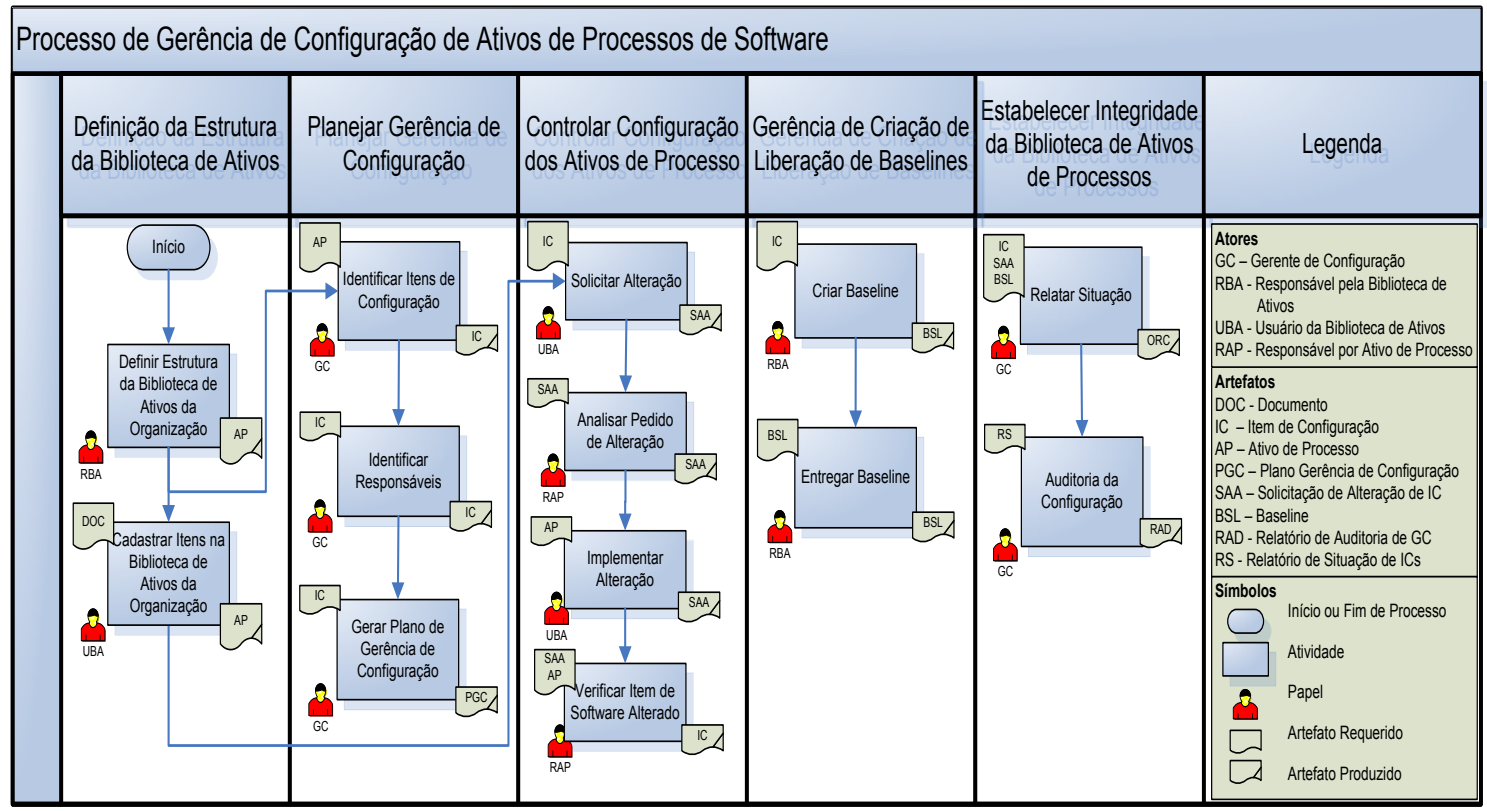

Figura 1 - Processo Integrado para Gerência de Ativos de Processos

O primeiro passo desse processo envolve Definir Estrutura da Biblioteca de Ativos da Organização seguindo um esquema considerado adequado pela organização para o armazenamento dos ativos. Para cada item desta estrutura pode-se definir subitens (como em uma estrutura de pastas do sistema operacional), os tipos de ativos de processo que devem ser armazenados (por exemplo, processos, política organizacional etc.) e também as ferramentas que podem ser úteis para criar, editar ou gerenciar tais ativos. Cada um dos diferentes tipos ativos de processo podem ter roteiros associados que auxiliam a geração dos documentos. Após feita a definição da estrutura, é possível Cadastrar Itens na Biblioteca de Ativos da Organização, dessa forma, instâncias dos ativos de processos da organização de forma a armazená-las e possibilitar, se for o caso, a gerência de configuração. Os ativos de processo possuem um conjunto de estados aos quais podem ser associados: não iniciado, em execução, finalizado, avaliado e aprovado.

Os itens presentes na Biblioteca de Ativos não são automaticamente alvo de gerência de configuração. Para que isso ocorra, é preciso, primeiro, fazer um plano de Gerência de Configuração que os incluam. O primeiro passo desse planejamento é Identificar Itens de Configuração que ficarão sob controle da gerência de configuração, ou seja, os produtos de trabalho que compõem cada item de configuração. Cada item ficará sob controle da gerência de configuração a partir do momento em que o documento submetido à biblioteca for considerado aprovado pela primeira vez. Deve-se também Identificar Responsáveis tanto pelas atividades do processo de gerência de configuração quanto por cada item de configuração individualmente. As responsabilidades sobre os itens de configuração envolvem aprovar as solicitações de alteração e as alterações nos itens de configuração. Na identificação da configuração é possível selecionar os responsáveis por cada item de configuração dentre os responsáveis que foram identificados neste momento. Cada item poderá possuir diferentes responsáveis de acordo com suas características. De posse destas informações é possível, de fato, Gerar Plano de Gerência de Configuração, que contém as 
atividades relacionadas ao processo de gerência de configuração, assim como os responsáveis por estas atividades.

Após um documento ter sido incluído na biblioteca de ativos e considerado aprovado as alterações passarão a ser controladas via um subprocesso cujo objetivo é manter o controle e o registro das mudanças realizadas nos itens de configuração ao longo do tempo. A primeira atividade deste subprocesso é Solicitar Alteração em um ativo de processo, que consiste em elaborar um pedido de alteração num item de configuração justificando a sua necessidade e impacto que esta modificação irá provocar, como por exemplo, pode ser necessário alterar outros itens após modificar este item para que os itens fiquem consistentes. Este pedido deverá ser posteriormente aprovado pelos responsáveis por aquele item de configuração. Durante a execução da atividade Analisar Pedido de Alteração os responsáveis pelo item de configuração podem visualizar os pedidos de alteração realizados e, em seguida, aprovar ou não um determinado pedido de alteração que esteja pendente. Caso a alteração não seja aprovada o estado do item volta a ser liberado para que outras pessoas possam solicitar alterações, caso contrário é necessário indicar um responsável, que pode ser ou não o próprio autor do pedido, por Implementar Alteração. Se o pedido de alteração de um determinado item for aprovado, o responsável pela alteração poderá realizá-la. O primeiro passo é obter a última versão do item de configuração a ser alterado e então, após realizar as alterações indicadas, enviar o documento modificado para análise aos responsáveis pelo item de configuração informando as modificações efetivamente realizadas. Por fim, um dos responsáveis pelo item de configuração deve Verificar Item de Software Alterado após visualizar os itens de configuração que foram alterados e analisar a alteração de um determinado item de configuração para que se possa fornecer um parecer. Três situações diferentes podem ocorrer de acordo com os pareceres (i) não aprovado: neste caso nada é feito em relação à última versão do item de configuração alterado, que volta a estar liberado para que outras pessoas o modifiquem desde que tenham seu pedido de alteração aprovado; (ii) aprovado: neste caso é gerada uma nova versão do item de configuração em questão contendo a alteração implementada, assim, esta passa a ser a última versão disponível deste item e o item de configuração vai para a situação de liberado; e (iii) rever alterações: neste caso o responsável pela alteração é notificado que deve rever alguns fatos na alteração para que a mesma seja aprovada, após estas modificações terem sido efetuadas, o item alterado deve ser novamente enviado para análise e a atividade de verificação do item de software alterado deve ser executada novamente.

Uma baseline é uma versão formalmente aprovada de um item de configuração, independente de mídia, formalmente definida e fixada em um determinado momento durante o ciclo de vida do item de configuração [SOFTEX 2007b]. Periodicamente, deve-se Criar Baseline de forma a controlar a liberação e a entrega dos ativos de processos levando em consideração as políticas da organização e as necessidades identificadas pelos responsáveis pelos processos e demais ativos de processos. Neste momento, deve-se identificar os itens de configuração e as versões destes itens que compõem uma baseline e descrever esta baseline além das diferenças para com a anterior. Após criada, é possível Entregar Baseline aos interessados. Para isso, o responsável pelos ativos de processo deve em primeiro lugar selecionar a baseline a ser entregue, empacotá-la e em seguida entregá-la ao receptor, por exemplo, através da disponibilização em uma intranet ou diretamente através de e-mail. 
A atividade Relatar Situação tem por objetivo manter todos os envolvidos no projeto informados sobre as alterações ocorridas nos itens de configuração. Logo, informações como quem modificou um certo item, quem está modificando determinado item, quando uma modificação ocorreu, quais itens serão afetados por uma alteração, porque uma alteração foi necessária, quais são as versões de itens, quais baselines foram criadas, entre outras questões, devem ser mantidas. $\mathrm{O}$ acesso a estas informações deve ser rápido. Este relatório pode ser útil para divulgar modificações realizadas em um determinado ativo de processo e, também, durante a Auditoria da Configuração. Esta atividade visa assegurar que as alterações tenham sido implementadas corretamente e analisar a integridade dos itens de configuração e baselines. Essas auditorias são conduzidas de acordo com processos bem definidos que se constituem de vários papéis e responsabilidades de auditores. Logo, cada auditoria deve ser planejada cuidadosamente. Uma auditoria pode necessitar de certo número de indivíduos para realizar uma variedade de tarefas durante um período de tempo razoavelmente curto.

O processo descrito não é completamente executado, de fato, seqüencialmente. A definição da estrutura da biblioteca de ativos deve ser executada num momento inicial e, logo após, deve-se cadastrar os itens na biblioteca. A partir deste momento a definição do plano de gerência de configuração pode ser realizada e, posteriormente, verificada sempre que necessário. As demais atividades podem ser executadas várias vezes ao longo do tempo, por exemplo, sempre que um usuário da biblioteca de ativos necessitar alterar um determinado ativo de processo.

\section{Outras Abordagens}

O escopo do apoio ferramental para auxiliar na execução de iniciativas de melhoria de processos em organizações encontrados na literatura é variado. Um dos apoios relatados é o feito em relação ao processo de gerência de configuração ou a criação e gerência de uma biblioteca de ativos de processos.

O SIR-CM [Park et al. 2007] é uma ferramenta de gerência de ativos de processo envolvidos em uma iniciativa de melhoria de processos de software. Esta ferramenta é um repositório que pode armazenar e gerenciar ativos heterogêneos e variados de processos de software. Permite a visualização de dados históricos e possui funcionalidades para disponibilização de métricas e gerência de documentos.

O e-R\&D [Ebert e De Man 2002] é um sistema de gerência de workflow e adaptação de processos com uma biblioteca de ativos com apoio de gerência de configuração utilizado para gerenciar a diversidade de processos dentro de uma corporação francesa. Segundo os autores, os benefícios conseguidos incluíram: melhoria da qualidade, redução do tempo de desenvolvimento, aumento da flexibilidade de engenharia, redução de esforço para tarefas, melhoria na comunicação, melhoria no alinhamento de processos e ferramentas, maior facilidade de geração de planos de treinamento.

WAGNER EPG/ER [Kurniawati e Jeffery 2004] é utilizado para a divulgação de processos numa organização através de um guia eletrônico de processos (EPG, do inglês Eletronic Process Guide) e baseado no framework WAGNER com apoio de um repositório de experiências (ER). O objetivo do sistema é divulgar os processos da organização, aumentando o seu uso e melhorando a institucionalização, além da criação 
de um repositório de experiências e, conseqüentemente, do aumento da memória organizacional.

Dentre as abordagens estritamente comerciais, pode citar o Microsoft Solutions Framework for CMMI Process Improvement que encapsula uma abordagem para o desenvolvimento formal de software e melhoria contínua do processo [Hundhausen 2006]. Este framework é composto por um guia com processos básicos associados com papéis comuns no desenvolvimento de software (por exemplo, gerentes, testadores, desenvolvedores etc.) e templates que auxiliam a executar as tarefas destes processos. As ferramentas disponíveis no Visual Studio Team System (VSTS) [Microsoft 2008] podem ser utilizadas em conjunto com este framework. Em relação à gerência de configuração, a ferramenta VSTS provê sistema de controle de versão, criação de baselines diretamente relacionados a código e artefatos de engenharia de software e gerenciamento do fluxo de mudança nos itens de configuração; o SharePoint [Microsoft 2007] provê versionamento mais simples e armazenamento de artefatos (documentos principalmente) com controle de acesso. Estas ferramentas, no entanto, precisam ser configuradas adequadamente para uso e devem ser adaptadas ao processo em uso e ao fluxo de vida dos produtos de trabalho em cada organização individualmente. Outras ferramentas de gerência de configuração como o IBM Rational ClearQuest [IBM 2008], CVS [Ximbiot 2008] e SVN [Tigris 2008] também podem ser utilizadas como repositórios de arquivos e funcionar como uma biblioteca de ativos organizacionais.

\section{Estudo de Caso}

Em janeiro de 2007 a Organização A começou a implementação de processos visando a uma avaliação no Nível F do MPS.BR. Esta organização está localizada no Rio de Janeiro e é especializada no desenvolvimento e comercialização de pacotes de soluções, principalmente para os ramos da saúde, educação e finanças. O principal objetivo de qualidade do programa de melhoria de processos de software da empresa foi o aumento da qualidade dos produtos e a diminuição do esforço de manutenção, devido à longa vida útil e adaptabilidade de seus produtos. Em outubro de 2007 a Organização A obteve o Nível F do MPS.BR.

Apesar de o Nível F do MPS.BR não prever a definição de processos padrão fez parte da estratégia adotada a definição de um processo padrão para Desenvolvimento de Software além de processos padrão referente aos processos Gerência de Projetos, Gerência de Requisitos, Garantia da Qualidade, Gerência de Configuração e Medição, previstos pelo MPS.BR. Além destes processos, outros ativos organizacionais foram identificados: documento de nomeação do Grupo de Processos (que envolve os membros do Grupo de Garantia de Qualidade, Gerência de Configuração e Medição), definição da política organizacional, plano de gerência de configuração organizacional e plano de medição organizacional. Uma estrutura compatível com estes ativos de processos foi definida na Estação Taba, como pode ser vista na Figura 2. Foi definida uma estrutura adequada para armazenar: (i) Política Organizacional; (ii) Nomeação do Grupo de Processos; (iii) Processo de Desenvolvimento; (iv) Processos (referentes a cada processo do Nível F do MPS.BR); (v) Plano de Gerência de Configuração Organizacional; e (vi) Plano de Medição e Análise Organizacional. 


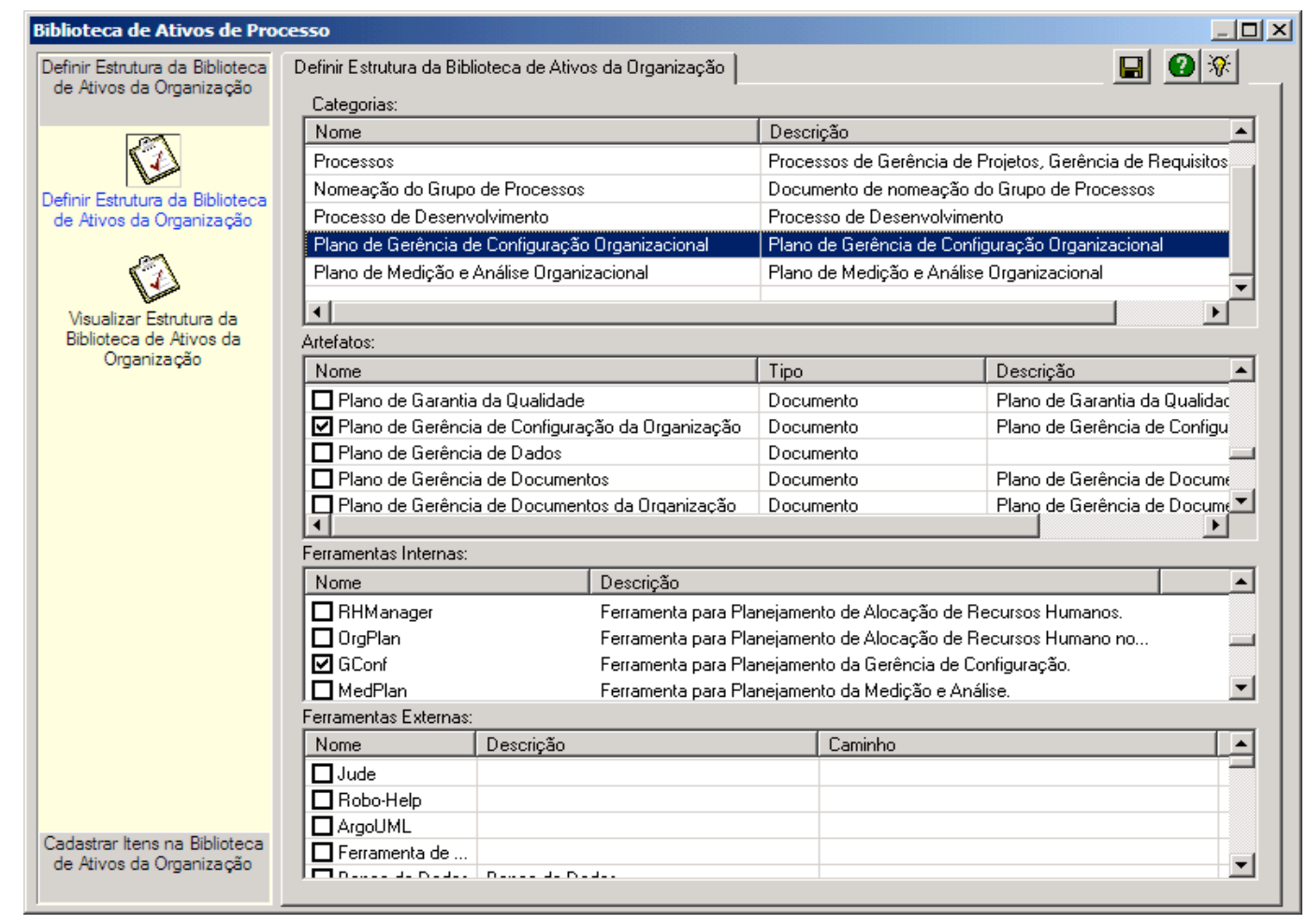

Figura 2 - Definição de Processos para a Organização

A Figura 2 mostra a tela da ferramenta GConf responsável por elaborar o plano de gerência de configuração. Pode-se ver, nesta tela, a escolha dos itens da Biblioteca de Ativos que devem ser gerenciados. Note no lado direito da Figura as atividades do processo descrito na seção anterior (Definir Estrutura da Biblioteca de Ativos da Organização e Visualizar Estrutura da Biblioteca de Ativos da Organização).

A figura 3 exibe a tela da ferramenta GConf que apóia o planejamento da Gerência de Configuração. O objetivo desta atividade é permitir que o Gerente de Configuração identifique os itens de configuração que terão suas alterações controladas em um determinada granularidade. Para os itens de configuração presentes em uma baseline, será necessário solicitar a alteração, ter a solicitação aprovada, alterar o item e ter a alteração aprovada. Em seguida, será gerada automaticamente uma nova versão do item de configuração referente ao artefato alterado. Para os itens de configuração que não estão presentes em nenhuma baseline, basta informar a alteração a ser realizada. Neste caso, uma nova versão do item de configuração também é gerada. 


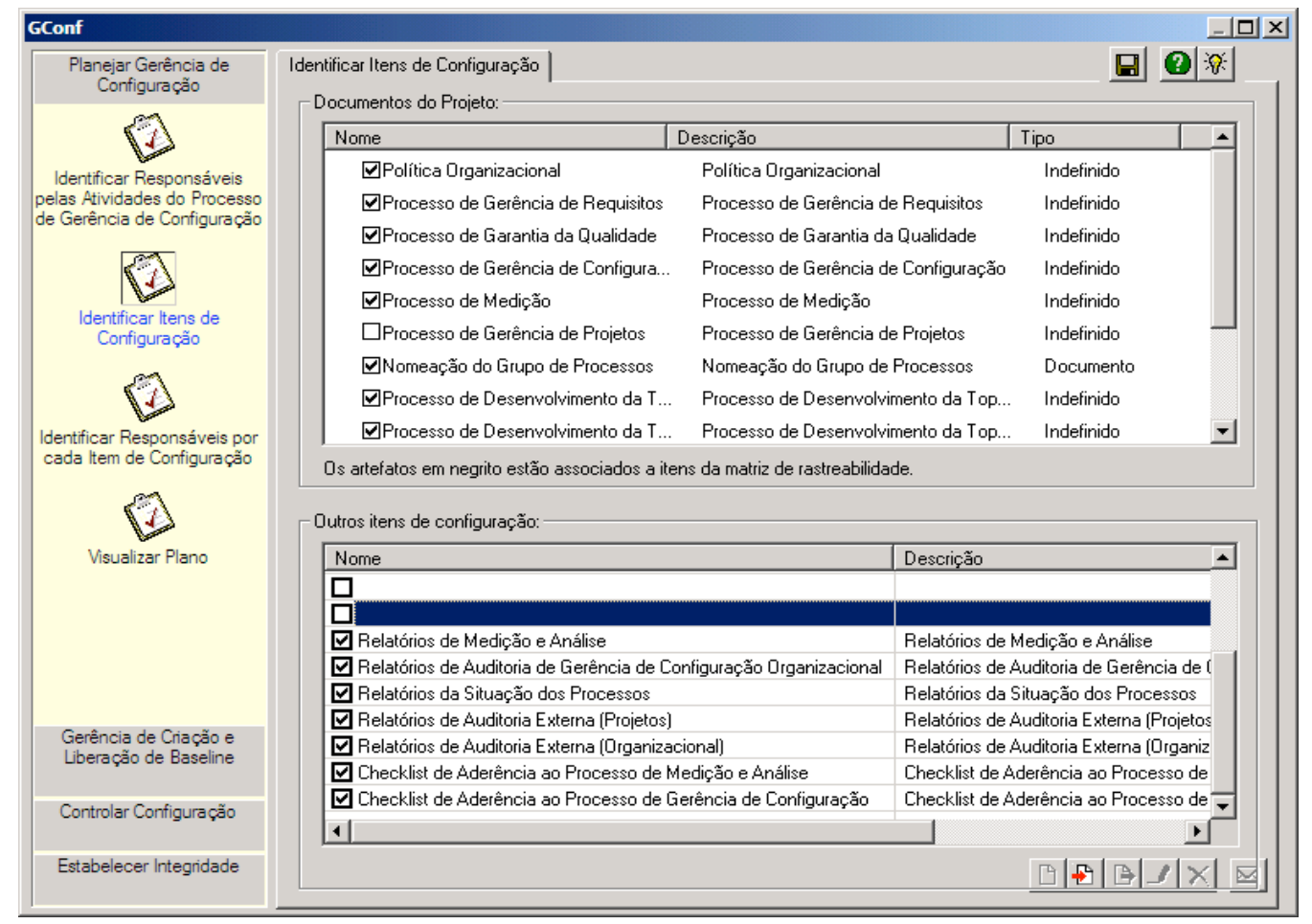

Figura 3 - Definição do Plano de Configuração de Ativos de Processos

A Figura 4 exibe a tela da ferramenta onde pode ser feito o cadastro das instâncias dos ativos de processos. Note que o item selecionado (Plano de Gerência de Configuração da Organização) é um item de configuração presente no Plano de Gerência de Configuração e está com a situação "Aprovado", dessa forma, a alteração deste ativo de processo só pode ser feita com a utilização da ferramenta GConf.

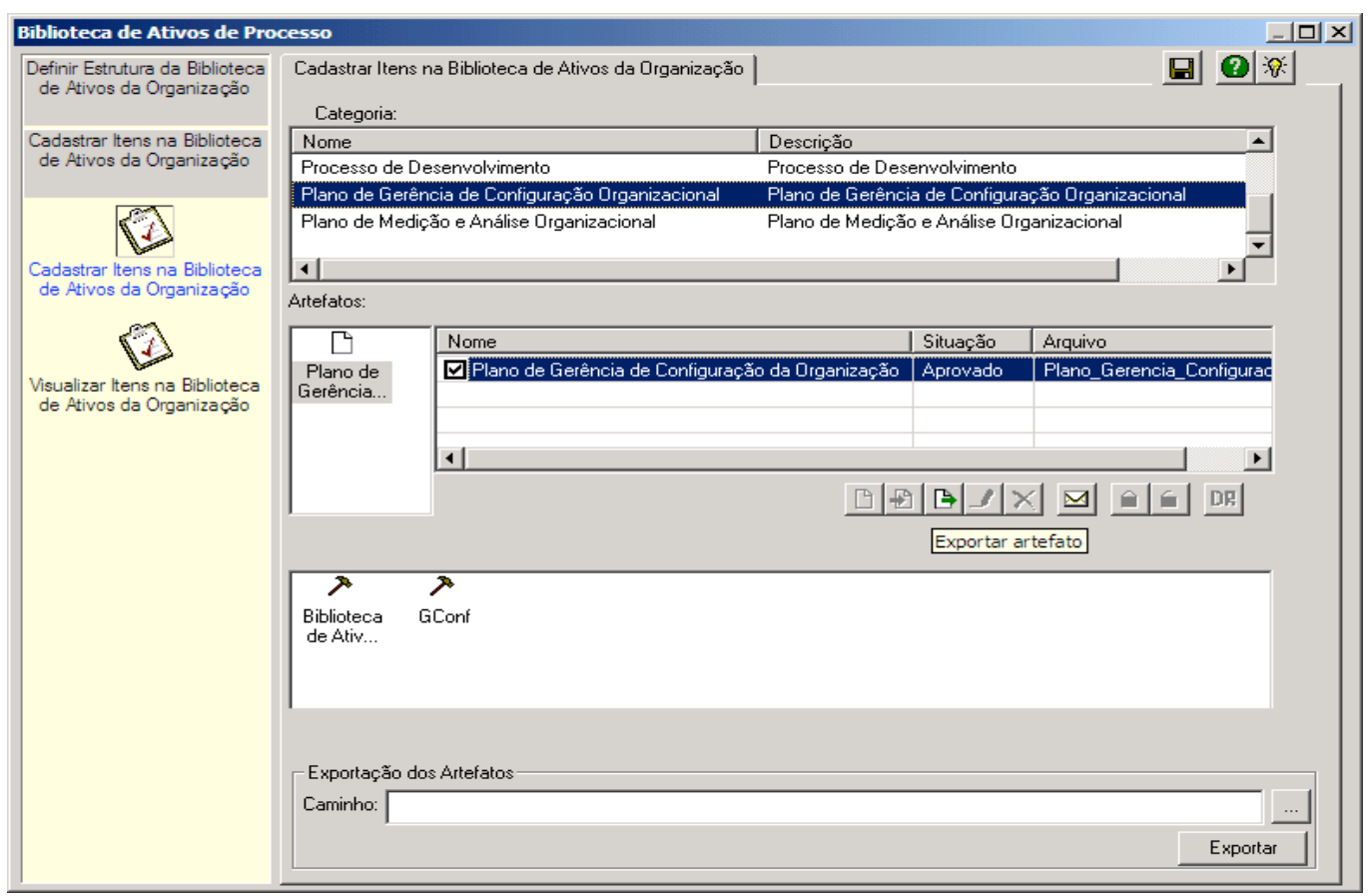

Figura 4 - Cadastro de Ativos de Processo 
A Figura 5 apresenta a tela de análise do pedido de alteração sob um ativo de processo. Neste caso, o Processo de Medição.

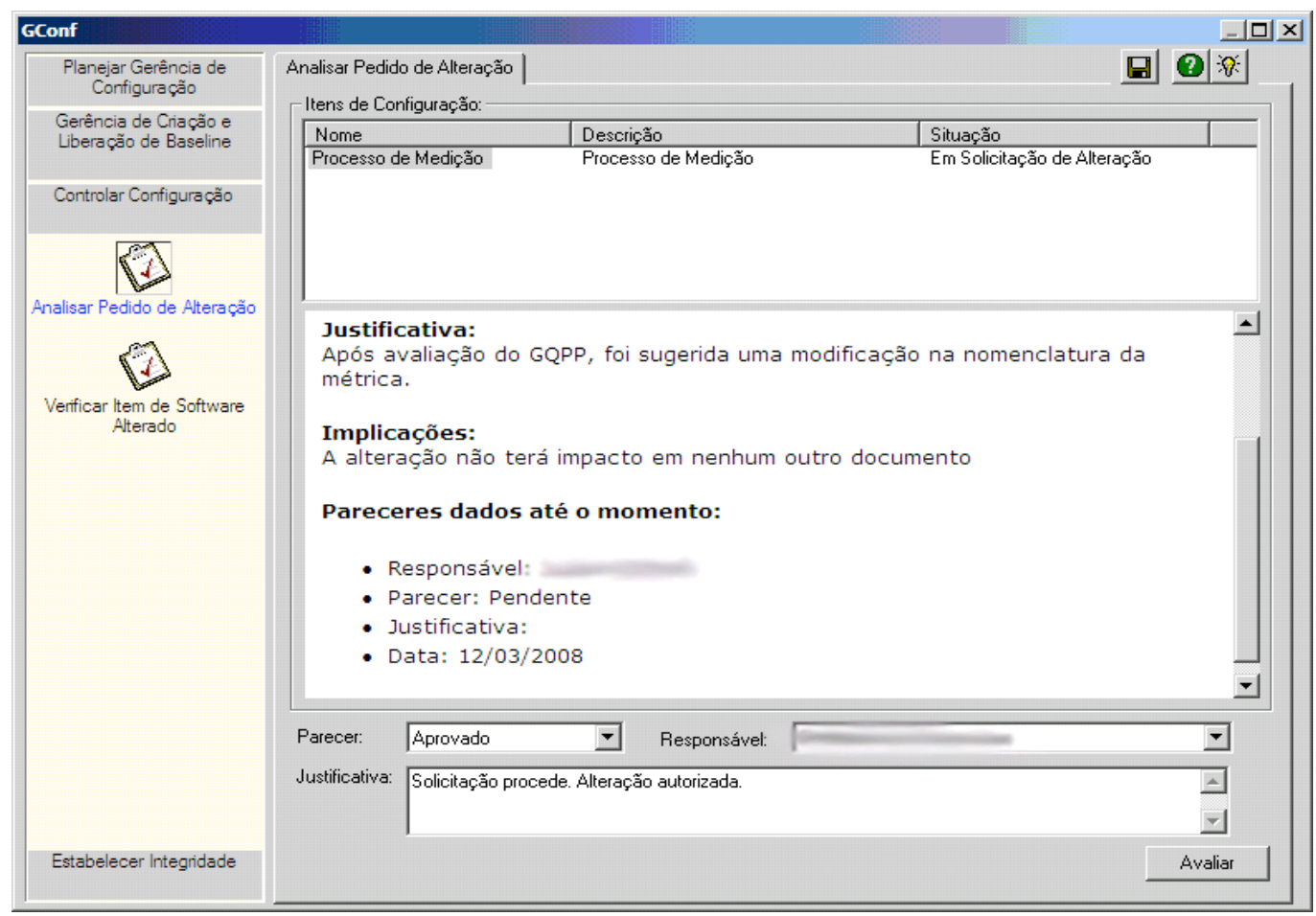

Figura 5 - Alteração de Ativo de Processo sob Gerência de Configuração

Antes da avaliação na empresa foi criada uma baseline dos ativos organizacionais contendo: Nomeação do Grupo de Processos, Plano de Gerência de Configuração da Organização, Plano de Medição da Organização, Política Organizacional, Processo de Desenvolvimento, Processo de Garantia da Qualidade, Processo de Gerência de Configuração, Gerência de Projetos, Processo de Gerência de Requisitos, Processo de Medição. Devido a uma alteração no Plano de Medição da Organização os seguintes documentos necessitaram ser alterados, também: Processo de Desenvolvimento, Processo de Garantia da Qualidade, Processo de Gerência de Configuração, Gerência de Projetos, Processo de Gerência de Requisitos, Processo de Medição. Um outro documento, Nomeação do Grupo de Processos, também foi alterado devido a modificações na estrutura e participantes do Grupo de Processos. Todos os registros foram mantidos pela ferramenta GConf e o processo de alteração implementado por ela foi seguido.

De posse destes documentos e dos registros na forma de um relatório gerado automaticamente, foi possível realizar uma auditoria no conteúdo da biblioteca de ativos antes da avaliação para evidenciar que a integridade das baselines dos documentos organizacionais era estabelecida e mantida através de auditoria da configuração e de registros da Gerência de Configuração.

O uso da abordagem descrita permitiu a gerência e controle dos ativos de processos e também facilitou a auditoria de configuração sob o conteúdo da biblioteca de ativos. Atualmente a Organização A está definindo uma nova versão dos processos de forma a atender toda a organização, englobando projetos de customização, integração e migração, além dos projetos de desenvolvimento. Devido a essa nova definição do 
processo de desenvolvimento será necessário, também, alterar os demais processos, o que deve ser feito com o auxílio das ferramentas já utilizadas.

\section{Perspectivas Futuras}

O foco principal das ferramentas é a integração com as outras ferramentas da Estação Taba visando a automatização do maior número possível de tarefas do engenheiro de software com o maior nível de qualidade possível. O objetivo é diminuir as dificuldades inerentes do ciclo de vida de um software.

O ponto principal da abordagem que deseja-se tornar transparente para o engenheiro de software é a atividade de auditoria da configuração. Atualmente, a abordagem apóia a execução desta atividade com informações sobre os itens de configuração e os baselines. Então, o auditor deve analisar estas informações a fim de avaliar os itens de configuração e os baselines em relação a critérios pré-estabelecidos pela organização. A nova proposta, que ainda será implementada, deve tornar a avaliação destes critérios automatizada, de modo que o engenheiro de software não utilize seu tempo avaliando os critérios que a ferramenta pode avaliar automaticamente. Como exemplo de critérios que poderão ser avaliados automaticamente pode-se citar: itens de configuração com pedidos de alteração pendentes a mais de $\mathrm{X}$ dias, itens de configuração que estão em alteração a mais de $\mathrm{X}$ dias, itens de configuração que deveriam estar na baseline mas não estão, artefatos que deveriam ter sido gerados mas que não foram e artefatos que deveriam estar sob controle da gerência de configuração mas que não estão. $\mathrm{O}$ auditor poderá escolher quais critérios devem estar presentes nas avaliações.

Um outro esforço de melhoria a ser realizado é a integração da ferramenta de gerência de configuração e da biblioteca de ativos com ferramentas de gerência de configuração existentes no mercado, como por exemplo o cvs [CVS 2006], source safe [Microsoft 2008], e o subversion [Rooney 2005]. Normalmente as empresas já utilizam alguma ferramenta de gerência de configuração e acredita-se que a integração das ferramentas descritas neste artigo com as ferramentas utilizadas nas empresas possa facilitar a utilização da abordagem. Com a modificação a ser realizada, o engenheiro de software poderá efetuar controlar os pedidos de alteração dos itens de configuração e efetuar as auditorias através da Estaçao Taba e a Estação Taba delegaria para uma ferramenta de gerência de configuração externa o controle de versões dos itens de configuração e dos baselines, de forma transparante.

\section{Considerações Finais}

Este artigo apresentou a estratégia de Gerência de Configuração de Ativos de Processos de Software em uso na Estação Taba. Apresentou também o estudo de caso de utilização dessa estratégia e, também, das duas ferramentas que a implementam numa empresa de software no Rio de Janeiro visando a criação de uma biblioteca de ativos de processos utilizados durante a implementação de processos que culminou com uma avaliação no Nível F do MPS.BR.

Atualmente a Estação Taba está sendo evoluída para apoiar a definição e construção de Ambientes de Engenharia de Software Orientados a Corporação [Santos 2005] visando prover o apoio computacional que possibilite a uma corporação, em relação aos processos de software, gerenciar a diversidade e os estágios de maturidade 
de cada uma das organizações que a compõem de forma adequada às suas necessidades. Do mesmo modo que a diversidade de processos no contexto corporativo é maior do que em uma organização isolada, a quantidade de ativos de processo relacionados a estes processos também é maior e também devem ser gerenciados e controlados. Os Ambientes Orientados a Corporação devem, portanto, possuir uma biblioteca de ativos de processos que garanta à corporação a gerência dos seus ativos de processos e, além disso, permitir à corporação um maior controle sobre a gerência e evolução do conteúdo das bibliotecas de ativos de processos de suas organizações subordinadas. Dessa forma, a Biblioteca de Ativos de Processos com apoio de Gerência de Configuração descrita neste artigo será alterada para permitir esse novo tipo de controle.

Além disso, uma nova implementação da Estação Taba está sendo desenvolvida na Web. As ferramentas atuais serão migradas gradativamente. Dessa forma, uma nova implementação da Biblioteca de Ativos de Processo está prevista para 2008. Esta nova implementação, além dos benefícios que uma ferramenta Web proporciona, também abrangerá as melhorias descritas na seção anterior.

\section{Referências}

ANDRADE, J.M.S. (2005), Avaliação de Processos de Software em ADSOrg, Dissertação de M. Sc., COPPE, UFRJ, Rio de Janeiro, Brasil.

Baddoo, N., Hall, T. (2003), "De-motivators for software process improvement: An analysis of practitioners' views", Journal of Systems and Software, v. 66, n. 1, pp. 23 33.

Berger, P. (2003), Instanciação de Processos de Software em Ambientes Configurados na Estação TABA, Dissertação de M. Sc., COPPE, UFRJ, Rio de Janeiro, Brasil.

Campos, F.B., Albuquerque, A.B., Andrade, J.M., et al. (2006), "Abordagem em Níveis para Avaliação e Melhoria de Processos de Software". In: V Simpósio Brasileiro de Qualidade de Software - SBQS 2006, pp. 100-114, Vila Velha - ES.

Chrissis, M.B., Konrad, M., Shrum, S. (2006), CMMI (Second Edition): Guidelines for Process Integration and Product Improvement, Addison Wesley Professional.

CVS - Concurrent Versions System (2006). In: http://www.nongnu.org/cvs/, accessed in $13 / 03 / 2008$.

Ebert, C., De Man, J. (2002), "e-R\&D - Effectively managing process diversity", v. 14, n. 1-4, pp. 73--91.

Ferreira, A.I.F., Santos, G., Cerqueira, R., et al. (2007), "Applying ISO 9001:2000, MPS.BR and CMMI to Achieve Software Process Maturity: BL Informatica's Pathway". In: 29th Int. Conference on Software Engineering (ICSE), pp. 642-651, Minneapolis, USA, May.

Ferreira, A.I.F., Santos, G., Cerqueira, R., et al. (2006), "Taba workstation: Supporting software process improvement initiatives based on software standards and maturity models". In: Lecture Notes in Computer Science (including subseries Lecture Notes in Artificial Intelligence and Lecture Notes in Bioinformatics), v. 4257 LNCS, pp. 207-218, Joensuu.

Figueiredo, S., Santos, G., Rocha, A.R. (2004), "Gerência de Configuração em Ambientes de Desenvolvimento de Software Orientados a Organização". In: III Simpósio Brasileiro de Qualidade de Software - SBQS 2004, Brasília - DF.

Hundhausen, R. (2006), Working with Microsoft Visual Studio 2005 Team System, 5th ed. Redmond, USA, Microsoft Press. 
IBM, "IBM Rational ClearQuest". In: http://www306.ibm.com/software/awdtools/clearquest/, accessed in 13/03/2008.

IEEE (1990), "Std 610.12", IEEE Standard Glossary of Software Engineering Terminology, v. Std 610.12.

ISO/IEC-12207 (1998), "Tecnologia de Informação - Processos de ciclo de vida de Software", ABNT - ASSOCIAÇÃO BRASILEIRA DE NORMAS TÉCNICAS, v. ISO/IEC-12207.

ISO/IEC-12207:Amd1 (2002), "Information Technology - Amendment 1 to ISO/IEC 12207", The International Organization for Standardization and the International Electrotechnical Commission, v. ISO/IEC-12207:Amd1.

ISO/IEC-12207:Amd2 (2004), "Information Technology - Amendment 2 to ISO/IEC 12207", The International Organization for Standardization and the International Electrotechnical Commission, v. ISO/IEC-12207:Amd2.

Kurniawati, F., Jeffery, R. (2004), "The long-term effects of an EPG/ER in a small software organisation". In: Proceedings of the Australian Software Engineering Conference, ASWEC, v. 2004, pp. 128-136, Melbourne, Vic.

Microsoft, "Microsoft Office Sharepoint Server 2007". In: http://www.microsoft.com/sharepoint/default.mspx

Microsoft, "Visual Studio Team System 2005 Developer Center". In: http://msdn2.microsoft.com/en-us/teamsystem/default.aspx.

Microsoft Visual SourceSafe Roadmap (2008). In: http://msdn2.microsoft.com/enus/library/aa302175.aspx, accessed in 13/03/2008.

Montoni, M. (2003), Aquisição de Conhecimento: Uma Aplicação no Processo de Desenvolvimento de Software, Dissertação de M.Sc., COPPE, Dissertação, Rio de Janeiro, Rj, Brasil.

MONTONI, M., SANTOS, G., FIGUEIREDO, S., et al. (2006), "Uma Abordagem de Garantia de Qualidade de Processos e Produtos de Software com Apoio de Gerência de Conhecimento na Estação TABA". In: V Simpósio Brasileiro de Qualidade de Software - SBQS 2006, pp. 87-99, Vila Velha - ES.

Montoni, M., Santos, G., Rocha, A.R., et al. (2007), "MPS Model and TABA Workstation: Implementing Software Process Improvement Initiatives in Small Settings". In: Fifth Workshop on Software Quality held in conjunction with the 29th Int. Conference on Software Engineering (ICSE), Minneapolis, USA, May.

Niazi, M., Wilson, D., Zowghi, D. (2005), "A framework for assisting the design of effective software process improvement implementation strategies", Journal of Systems and Software, v. 78, n. 2, pp. 204-222.

Park, E.-J., Kim, H.-K., Lee, R.Y. (2007), "Frameworks of Integration Repository for Software Process Improvement using SOA". In: Computer and Information Science, 2007. ICIS 2007. 6th IEEE/ACIS International Conference on, pp. 200-206.

Rocha, A.R.C., Maldonado, J.C., Weber, K.C. (2001), Qualidade de Software: Teoria e Prática, 1a ed., Prentice Hall.

Rooney, G. (2005), Practical Subversion, ISBN 1590592905, 1a ed., paperback.

Santos, G. (2003), Representação da Distribuição do Conhecimento, Habilidades e Experiências através da Estrutura Organizacional, Dissertação de M. Sc., COPPE, UFRJ, Rio de Janeiro, Brasil.

Santos, G. (2005), Ambiente de Engenharia de Software Orientado a Corporações, Exame de Qualificação, COPPE, UFRJ, Rio de Janeiro, Brasil. 
Santos, G., Montoni, M., Figueiredo, S., et al. (2007), "SPI-KM - Lessons Learned from Applying a Software Process Improvement Strategy Supported by Knowledge Management", Product-Focused Software Process Improvement.

Santos, G., Villela, K., Montoni, M., et al. (2005), "Knowledge management in a software development environment to support software processes deployment", v. 3782 NAI, pp. 111-120, Kaiserslautern, Germany.

Silva Filho, R.C. (2006), Uma Abordagem para Avaliação de Propostas de Melhoria em Processos de Software, Dissertação de M.Sc., COPPE, UFRJ, Rio de Janeiro, RJ, Brasil.

SOFTEX (2007a), "MPS.BR - Guia de Implementação - Parte 3".

SOFTEX (2007b), "MPS.BR - Guia Geral".

SWEBOK, "Guide to the Software Engineering Body of Knowledge". In: www.swebok.org, accessed in 13/03/2008.

Tigris, "SubVersion". In: http://subversion.tigris.org/, accessed in 13/03/2008.

Villela, K. (2004), Definição e Construção de Ambientes de Desenvolvimento de Software Orientados à Organização, Tese de D. Sc., COPPE, UFRJ, Rio de Janeiro, Brasil.

VILLELA, K., SANTOS, G., MONTONI, M., et al. (2004), "Definição de Processos em Ambientes de Desenvolvimento de Software Orientados a Organização". In: III Simpósio Brasileiro de Qualidade de Software - SBQS 2004, pp. 4-18, Brasília - DF.

Ximbiot, "Ximbiot - CVS Wiki". In: http://ximbiot.com/cvs/wiki/, accessed in 13/03/2008.

Zaharan, S. (1998), Software Process Improvement - Practical Guidelines for Business Success, Addison-Wesley. 